\title{
Lossless 4D Medical Images Compression Using Adaptive Inter Slices Filtering
}

\author{
Leila Belhadef, Zoulikha Mekkakia Maaza
}

Department d'Informatique, Laboratoire SIMPA, Faculté des Mathématiques et d'Informatique, Université des Sciences et de la Technologie d'Oran Mohamed Boudiaf, USTO-MB, BP 1505, El Mnaouer, 31000 Oran, Algérie

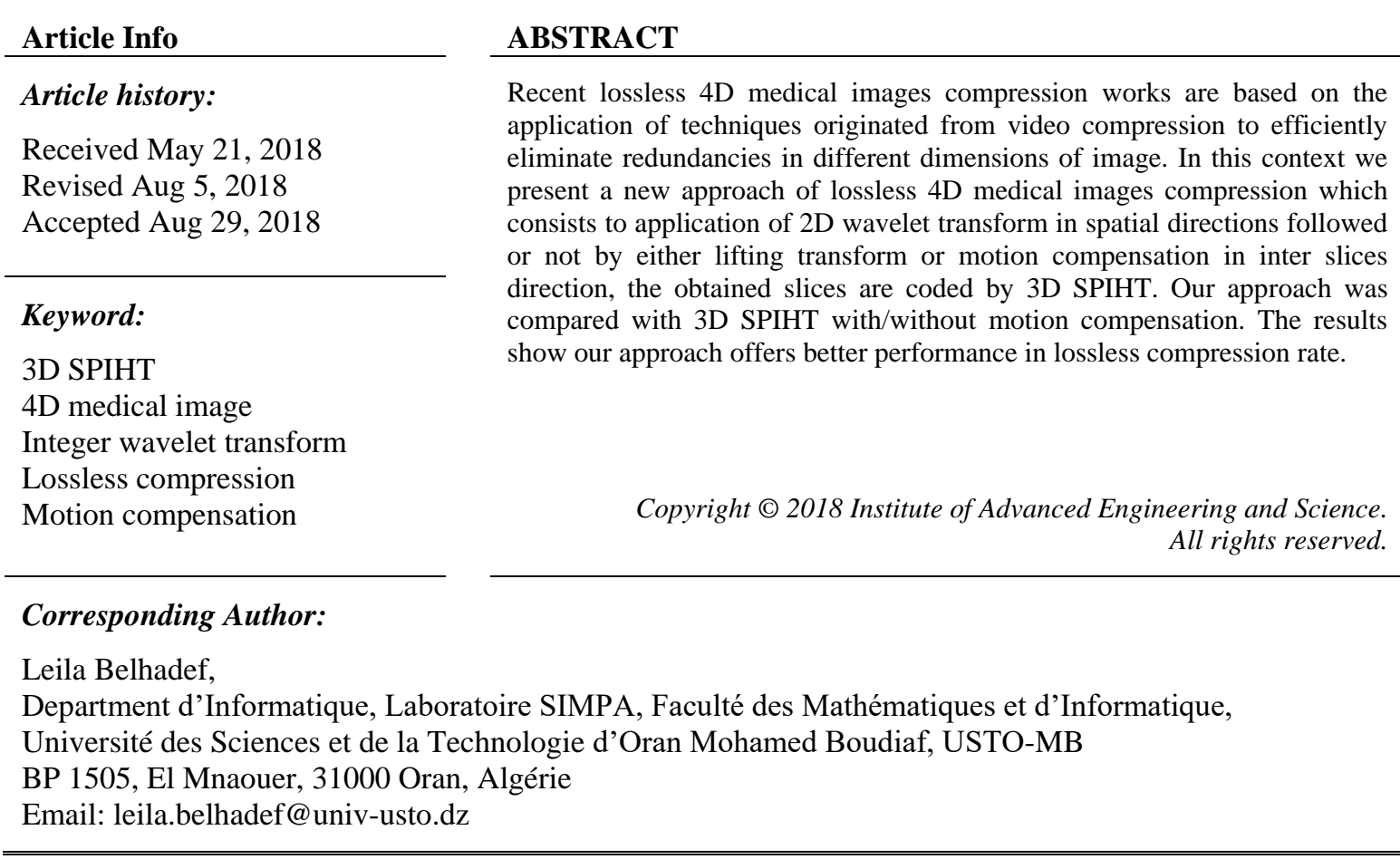

\section{INTRODUCTION}

4D medical images represent voluminous data where each image is composed by a set of volumes representing the 3D images of a human body part at different instants $\mathrm{t}$, each $3 \mathrm{D}$ image is in turn composed by a set of slices. In the literature a lot of lossless 4D medical images compression works are realized to reduce images size without data loss in order to avoid diagnosis errors. Considering 4D image as a set of volumes evolving in time, the recent lossless 4D medical images compression techniques apply the techniques of video compression such as motion compensation in order to efficiently removing inter slices or volumes redundancies.

Based on the advanced video coding H.264/AVC Sanchez et al. [1] described two coding methods for lossless 4D medical images compression. The first method exploits the redundancies among the 2D slices in (each volume) third dimensionz. The second method exploits the similarities between the slices in the fourth dimension time $t$. Redundancies through the fourth dimension are more numerous than redundancies through the third dimension; therefore this is the second method which permitted better compression ratio.

For more exploit the redundancies in the four dimensions of medical images the same authors of the precedent work proposed a new compression technique based on H.264/AVC [2] as in precedent work. So in this compression technique multi-frame motion compensation is applied firstly in $z$ dimension to each volume. The obtained reference and residual slices are treated secondly also by multi-frame motion compensation in temporal dimension, thus the redundancies are reduced in $z$ and $t$ dimensions. The final residual slices and motion vectors are compressed by entropy coding.

Here multi-frame motion compensation applied to a set of $n$ slices consists to consider first slice as reference slice intra coded and $n-1$ slices as inter slices which are inter coded by variable block matching 
and multi-frame motion compensation where each block of inter slices can be predicted from multi reference slices in $z$ dimension in its first application and $t$ dimension in its second application.

Martin et al. proposed in [3] a new method of 4D medical images compression also based on the H.264 video coding where predicted slices are generated by using spatio-temporal reference slices from $z$ and temporal neighborhood, where differently to precedent technique which realizes prediction in $z$ dimension followed by prediction in $t$ dimension, this technique realizes spatio-temporal prediction where each block of inter slice can be predicted from multi reference slices in spatio-temporal neighborhood, this permits to obtain a good results in compression compared prediction in $z$ dimension or prediction in time dimension.

All the previous cited works (based on H.264 video coding) don't integrate progressive lossy to lossless decoding in their schemes. Other techniques apply wavelet transform indeed it can be combined with image compression technique as fractal to improve time coding [4] also wavelet transform is very effective in medical image and video compression thus a lot of works are proposed as in [5] the authors presented color video medical compression technique by using geometric wavelet in order to eliminate efficiently similarity in each frame composed the video sequence with good image quality but without exploiting the temporal redundancies.

The first proposed works based on wavelet transform in literature for lossless 4D medical images compression do not integrate motion compensation in compression scheme thus in [6] the authors compared three techniques using JPEG2000. The first technique consists to apply 2D wavelet transform to each slice composed the volumes and compress it separately by using JPEG2000. The second technique is represented by two variants: the first variant is application of $1 \mathrm{D}$ wavelet transform in $z$ dimension, the second variant is application of 1D wavelet transform in $t$ dimension and for both variants the obtained slices are compressed by JPEG2000. The third technique is application of 1D wavelet transform in $t$ dimension flowed by $1 \mathrm{D}$ wavelet transform in $z$ dimension the obtained slices are compressed by JPEG2000. The exploitation of temporal dimension in second and third technique provides better results in compression rate because the numerous similarities in temporal dimension.

Due to efficiency of the motion compensation in the video compression scheme to reduce temporal redundancies Kasim et al. [7] proposed a wavelets compression technique of 4D medical images based on motion compensation and integer wavelet transform, here the 4D image is compressed as a sequence of 3D images. This technique consists to achieve 3D motion compensation in order to eliminate efficiently similarities existing between volumes in temporal direction, after the resulting volumes are decorrelated by $3 \mathrm{D}$ integer wavelet transform. The obtained data are coded by 3D SPIHT. This method allows lossless coding and progressive lossy to lossless decoding.

The first step is motion estimation inter 3D images. Where a group of $n 3 \mathrm{D}$ images are represented by one key image (the first image in a group is compressed without motion compensation) and $n-1$ inter images. Each current image (at position $t$ ) to be predicted is divided in cubes for each cube it most similar cube in reference image (at position $t-1$ ) is found. The difference between the position of current cube and it most similar cube is motion vectors and the difference between those cubes generates the residual image.

Thus the results of this step are 3D key image, $n-13 \mathrm{D}$ residual images and corresponding motion vectors. To reconstruct the initial group of images, the key image and the corresponding motion vectors is used to obtain predicted image which permits to reconstruct the first inter image by adding the predicted image to the first residual image, the followed inter images at position $t$ are reconstructed as the first one by using inter image at position $t-1$ and corresponding motion vectors. The second step consists to apply $3 \mathrm{D}$ integer wavelet transform to key image and residual images and in final step the key and residual images are coded by using 3D SPIHT, also the motion vectors are entropy coded. This technique permits to realize lossless compression and progressive lossy to lossless decoding by transmission a part of bit stream of key images followed by a part of bit stream of each inter image this permits to improve progressively the image quality in decompression.

All cited works of 4D medical image compression try to eliminate the redundancies by using different prediction techniques in $t$ and $z$ dimensions either by applying motion compensation or by applying wavelet transform or by applying both motion compensation flowed by wavelet transform.

In this paper we propose a new lossless compression approach based on adapted filtering in inter slices direction to improve the elimination of redundancies in 4D medical images. This approach consists to apply 2D integer wavelet transform to each slices followed or not by either wavelet filter or motion compensation in inter slices direction. The obtained slices are coded with 3D SPIHT. The experimental results are compared to two approaches: 3D SPIHT using motion compensated temporal filter in inter slices direction and 3D SPIHT (without motion compensation), the obtained lossless compression rates show our approach outperforms two other approaches. The rest of the paper is organized as follows. The section 2 presents prediction techniques used in video compression. The section 3 details the proposed approach of compression. In the section 4 the 
experimental results for lossless compression are compared to other compression approaches. In final section conclusions are presented.

\section{MOTION COMPENSATION AND TEMPORAL FILTERING}

The principals video compression techniques used in 4D medical images compression are represented by two types of technique: H.264/AVC video coding and wavelet video coding. These techniques are based on motion compensation to eliminate the redundancies as in H.264/AVC coder the motion compensation is applied by using the block matching to attain the minimum prediction error which is the minimum difference between current block (to be predicted) and reference block in search window, also the reference blocks can be determined from multi reference images with variable block size.

The lossless 4D compression techniques based on H.264/AVC offer good compression rate but without permitting the progressive decoding, the alternative is the wavelet techniques which produce scalable flux and realize good decorrelation of signal as presented in [7] where the progressive decoding is obtained by reordering of bit stream, the progressive decoding can be also obtained by using temporal scalability but it is not used here cause the motion compensation and wavelet transform is achieved separately.

In order to obtain temporal scalability and efficiency eliminate temporal redundancies the recent wavelet video coding apply the wavelet transform in motion trajectory by using motion compensated temporal filtering (MCTF). In wavelet video coding the MCTF can be applied before spatial decorrelation with wavelet transform $(t+2 D)$ or after spatial decorrelation in wavelet domain (2D+t or in-band) flowed by entropy coding.

The first works in video compression [8], [9] integrated Haar motion compensated temporal filtering where the images are separated in even images and odd images, the even images are low pass filtered and the odd images are high pass filtered, the obtained low pass images are also separated in even images and odd images and filtered till the temporal level decomposition is done. The filtering is realized in temporal trajectory determined by motion compensation to reduce the energy in obtained high pass filtered images. With the emergence of lifting scheme for the calculation of the integer wavelet coefficients, other works were carried as in [10] the authors defined two filters: motion compensated Haar lifting filter and motion compensated 5/3 lifting filter; the use of motion compensated 5/3 lifting filter gave the best results.

The scheme lifting is achieved using two steps prediction and update: the prediction step retains difference between two samples of signal (high pass filtering) and the update step retains the average therefore an approximation of signal (low pass filtering).

In [11] the authors introduced the motion compensated truncated 5/3 lifting filter where only the prediction step of lifting is performed, thus each high pass filtered image is obtained by determining the prediction error from neighbor reference images forward and backward. We propose an adaptive prediction in order to ameliorate lossless 4D medical images compression rate, we apply either 5/3 truncated lifting filter or motion compensated, if one of them minimize prediction error in inter slices direction.

\section{PROPOSED COMPRESSION SCHEME}

The first step of our scheme is formation of GOS (Group Of Slices) from 4D medical image followed by application of 2D integer wavelet transform (2D IWT) in spatial directions $(x, y)$ of each slices, the second step is inter slices filtering of each GOS and the final step is coding of obtained slices, motion vectors and etiquettes. As shown in Figure 1 proposed compression scheme.

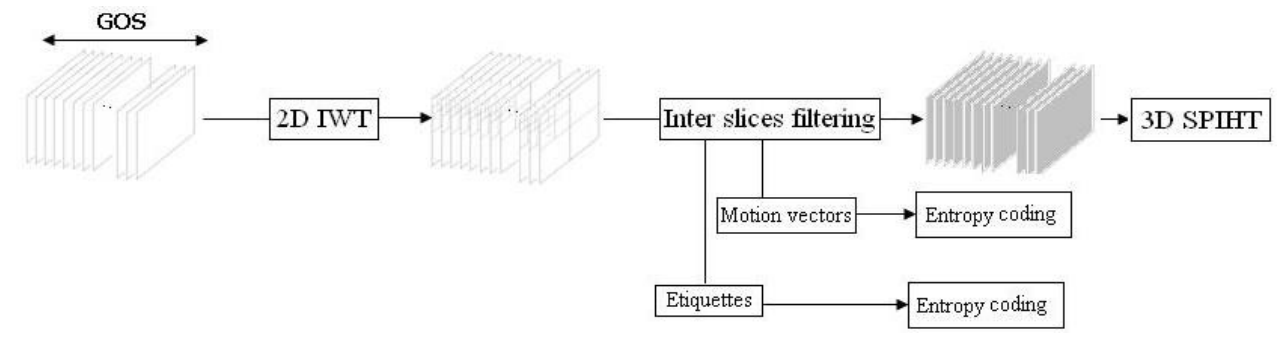

Figure 1. Proposed compression scheme

We describe these steps in the following points. 


\subsection{Construction of GOS and spatial transform}

The GOS are formed from the slices of 4D medical image $(x, y, z, t)$ as shows in Figure 2(a). This construction permits to obtain a set of GOS representing 4D medical image with each GOS is composed with nearest slices in spatial direction $z$ across time.

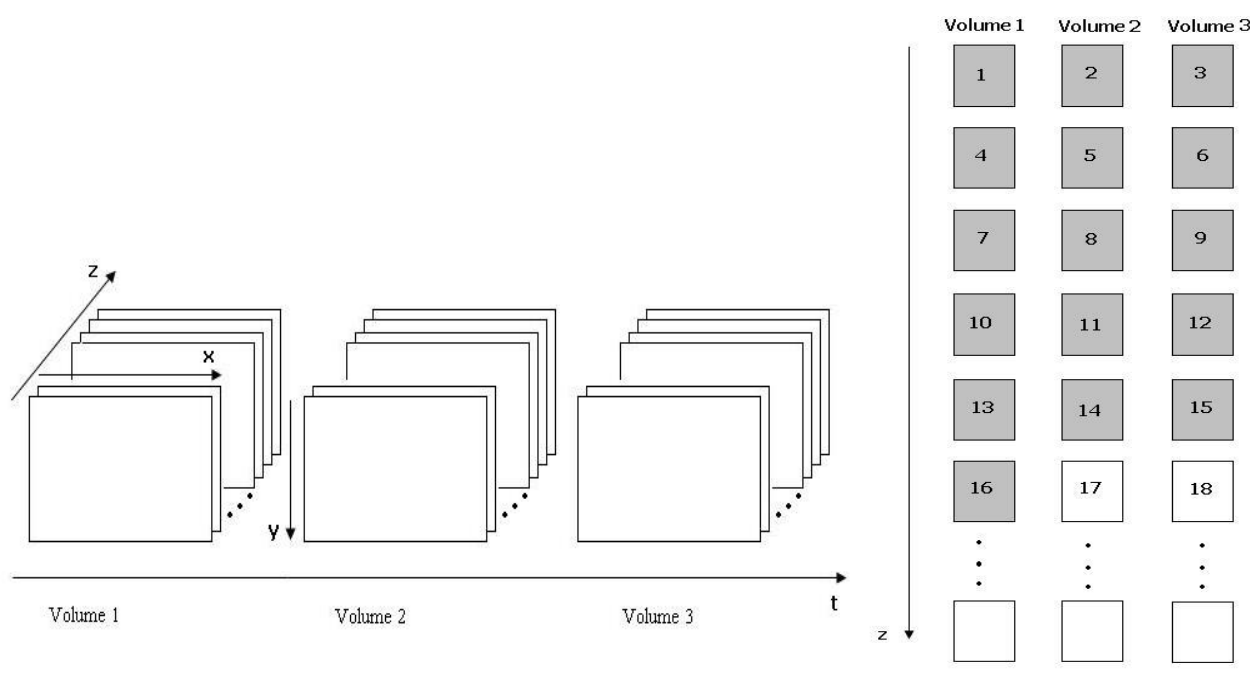

(a)

(b)

Figure 2. (a) 4D Image composed by 3 volumes. (b) Construction of GOS composed by 16 slices (in gray)

In Figure 2(b) the slices in gray represent the first GOS with 16 slices of 4D image composed by 3 volumes, thus all GOS of 4D image are also formed with follow slices in the same direction used for the first GOS (across time), as the second GOS starts with slice 17. Next the formation of GOS each slice is transformed by $2 \mathrm{D}$ integer wavelet transform using lifting scheme [12] in spatial directions $(x, y)$. This transform is reversible; it allows producing integer wavelet coefficients which permits lossless compression.

\subsection{Inter Slices Filtering}

After spatial transform each obtained GOS is filtered by the proposed filter in inter slices direction based on truncated lifting scheme and motion compensation. The motion compensated truncated 5/3 lifting filter proposed in [11] is realized by application of the truncated lifting scheme and motion compensation in the same time, the truncated lifting scheme achieves only prediction step of lifting scheme. The motion compensated truncated 5/3 lifting filter (MC Trunc 5/3) for the block $S_{k}[m, n]$ can be formulated as:

$R[m, n]=S_{k}[m, n]-\left\lfloor 0.5 \times\left(S_{k-1}\left[m-d_{1 m}, n-d_{1 n}\right]+S_{k+1}\left[m-d_{2 m}, n-d_{2 n}\right]\right)\right\rfloor$

Where $S_{k-1}, S_{k+1}:$ reference slices, and $S_{k}$ : inter slice.

$R$ : residual slice (high pass slice).

$\left(d_{l m}, d_{l n}\right)$ : motion vector of block $S_{k}[m, n]$ to a position in $S_{k-1}$.

$\left(d_{2 m}, d_{2 n}\right)$ : motion vector of block $S_{k}[m, n]$ to a position in $S_{k+1}$.

$\lfloor$.$\rfloor : corresponds to round operator.$

The motion compensation is realised by using block motion model so each inter slice (to be predicted) is divided in blocks and each block is filtered by MC Trunc 5/3. This filter achieves bi-directional motion compensation thus for each current block in $S_{k}$ it most similar block is determined in $S_{k-1}$ and in $S_{k+1}$ and the difference between the position of current block and these blocks represents motion vectors, the criteria of similarities used is minimum sum of absolute differences (SAD). Thus each inter slice is replaced by two motion fields as shows in Figure 3(a) and residual slice. 


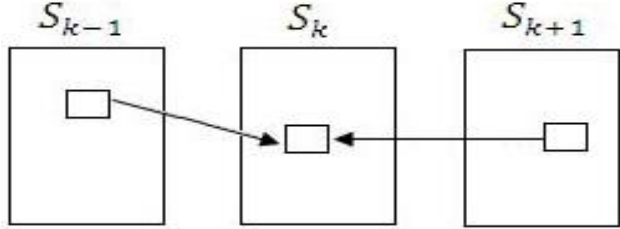

(a)

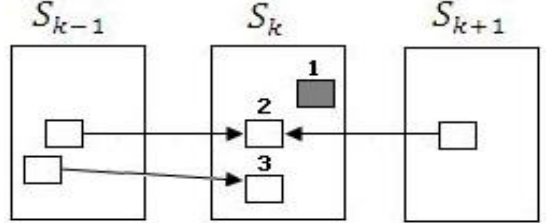

(b)

Figure 3. Motion fields: (a) predicted block with MC Trunc 5/3, (b) proposed approach with 1: unfiltered block $\left(E_{l}\right), 2$ : predicted block with truncated $5 / 3$ lifting transform $\left(E_{2}\right), 3$ : predicted block with motion compensated $\left(E_{3}\right)$

In our scheme each inter slice (to be predicted) is divided in block and each block is either unfiltered or filtered by one of following prediction techniques: Truncated 5/3 lifting transform or motion compensation. This filtering can be represented by three following prediction errors:

The unfiltered block $S_{k}[m, n]$ :

$$
E_{1}[m, n]=S_{k}[m, n]
$$

The truncated 5/3 lifting transform (without motion compensation):

$$
E_{2}[m, n]=S_{k}[m, n]-\left\lfloor 0.5 \times\left(S_{k-1}[m, n]+S_{k+1}[m, n]\right)\right\rfloor
$$

The motion compensated prediction:

$$
E_{3}[m, n]=S_{k}[m, n]-S_{k-1}\left[m-d_{m}, n-d_{n}\right]
$$

Where $\left(d_{m}, d_{n}\right)$ : motion vector of block $S_{k}[m, n]$ to a position in $S_{k-1}$.

Between the three prediction errors the minimum is chosen for determining the prediction error of this block $S_{k}[m, n]$ and its prediction method.

The prediction error $E_{1}$ represents the unfiltered block $S_{k}[m, n]$, the prediction error $E_{2}$ represents prediction step in 5/3 lifting transform and the prediction error $E_{3}$ is the difference between block $S_{k}[m, n]$ and it most similar block in slice $S_{k-1}$ as shows in Figure 3(b), the motion vector $\left(d_{m}, d_{n}\right)$ represents the displacement which allows minimizing $E_{3}$.

So each inter slice is replaced by one motion field (forward) and/or etiquette (to indicate which prediction error is used) and residual slice.

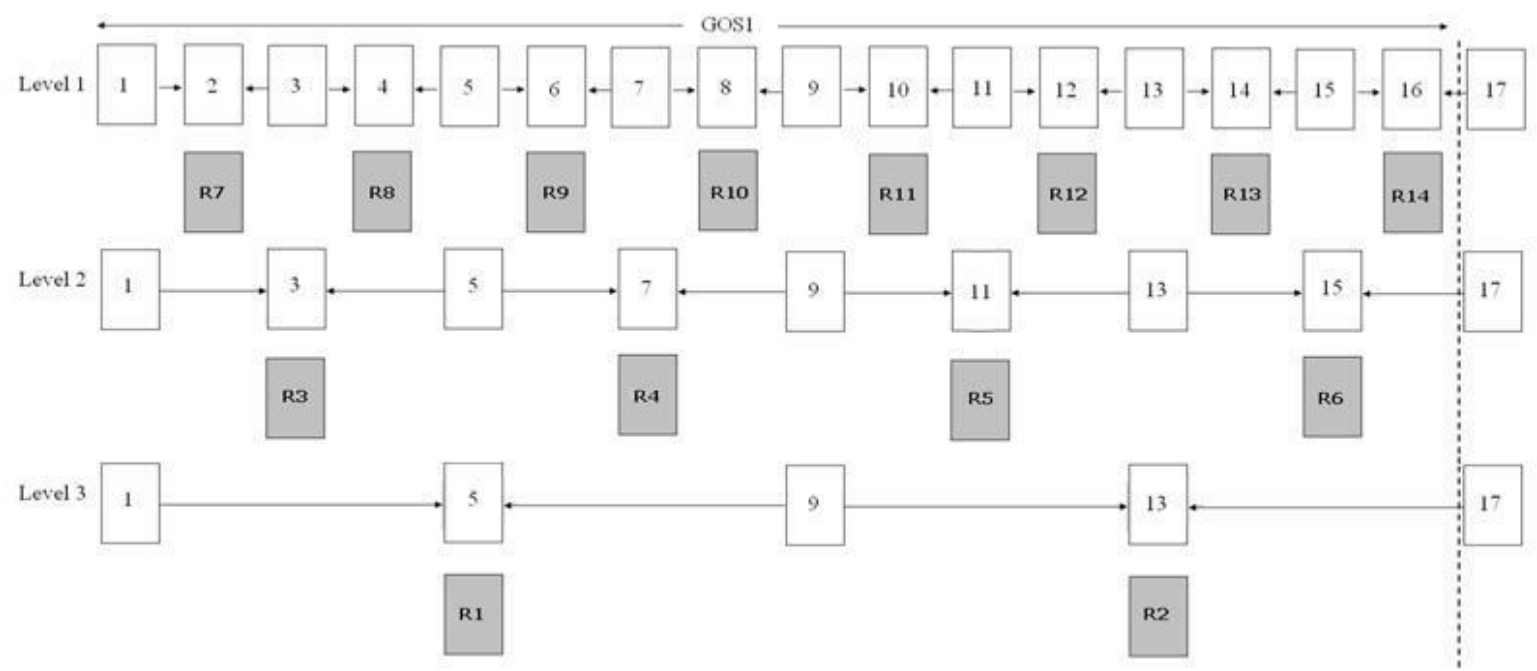

Figure 4. 3 levels motion compensated truncated 5/3 lifting filter for GOS with 16 slices 
Figure 4 presents 3 decomposition levels of MC Trunc 5/3 for GOS with 16 slices; the number of obtained slices of MC Trunc 5/3 is the same to our scheme which is composed by 2 reference slices $(1,9)$ and 14 residual slices (R1, R2, .. R14), the slice (17) represents the first reference slice in the next GOS.

However for motion coding, the approach with MC Trunc 5/3 produces two motion fields and our approach one motion field (forward) and etiquette for each predicted block by $E_{3}$ and only etiquette for each predicted block by $E_{1}$ or $E_{2}$.

\subsection{Reference and residual slices, motion vectors and etiquettes coding}

The obtained reference and residual slices composed GOS are coded by 3D SPIHT [13] (Set Partitioning in Hierarchical Trees) and generated bit stream is coded by arithmetic coder, 3D SPIHT is used as in video coding and volumetric image coding, it is a bit plan coder and it permits to reduce data size by exploiting the inter dependences of subbands in three dimensions $x, y$ and inter slices. Concerning the motion vectors and etiquettes are coded by arithmetic coder.

\section{EXPERIMENTAL RESULTS}

We tested the proposed compression method with five native 4D medical images CT (Computed Tomography) of heart from two references [14][15] shown in Figure 5. Each one is composed by 10 volumes and the spatial resolution of slices is $512 \times 512$ coded on 16bits per pixel. However the volumes of each 4D medical image have different sizes: data of reference [14] Image1 (141 slices/volume), Image2 (169 slices/volume) and Image3 (170 slices/volume) data of reference [15] Image4 (136 slices/volume) and Image5 (120 slices/volume).
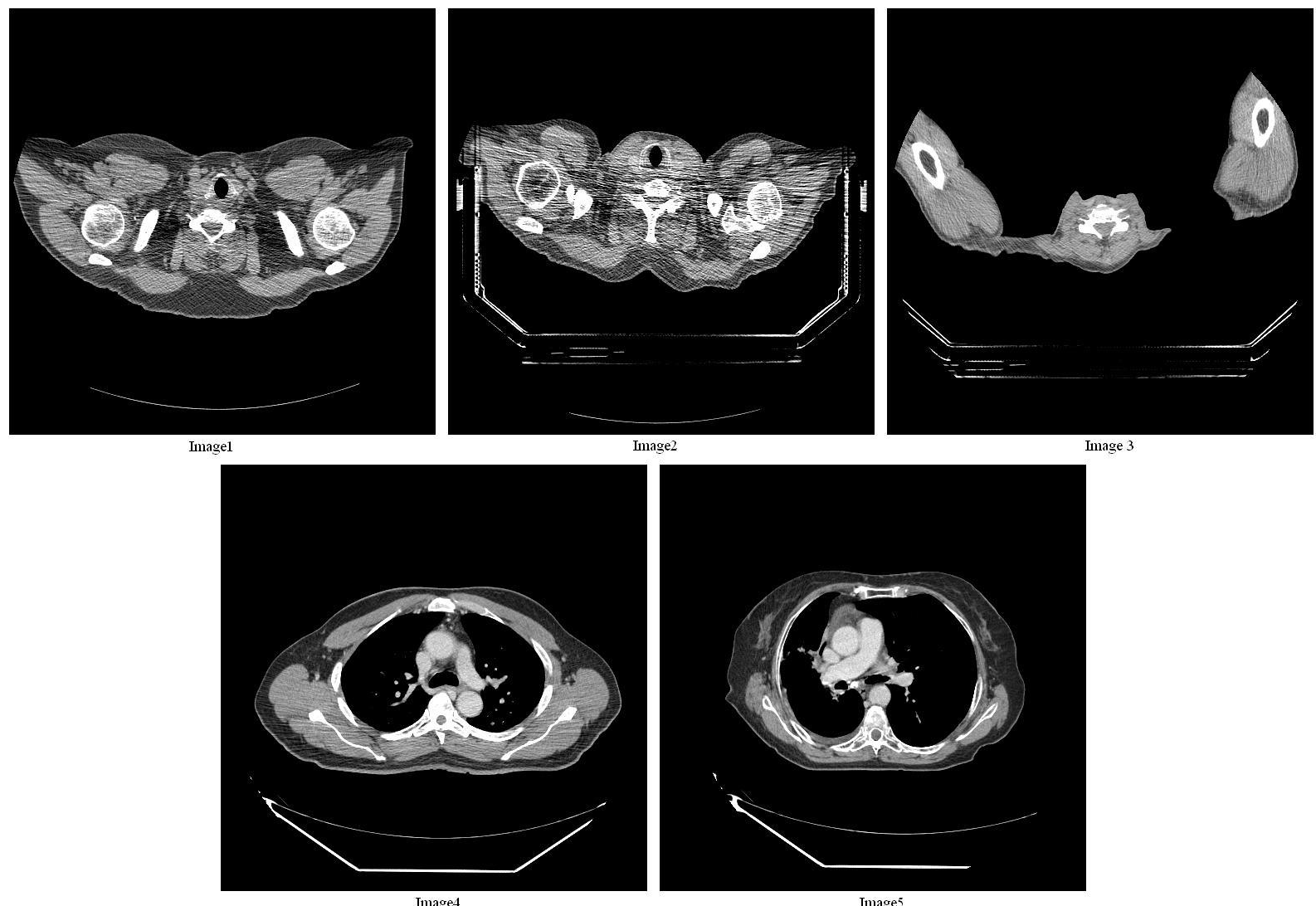

Image 3

Image4

Image5

Figure 5. First slices of each 4D medical image

We compare the experimental results of our approach with MC Trunc 5/3 approach (3D SPIHT with MC Trunc 5/3) and 3D SPIHT without motion compensation. For all these approaches GOS are composed by 16 slices formed as shows in Figure 2 (b). 
After each GOS is transformed with 3 levels 2D integer wavelet transform in spatial directions $(x, y)$ we use 5/3 filter. In inter slices direction of GOS our approach uses the proposed filter equations (2, 3 and 4), the MC Trunc 5/3 approach uses equation (1) with also three levels of decomposition for both. However 3D SPIHT without motion compensation uses in inter slices direction 1D integer wavelet transform with three levels of decomposition of the same used filter in spatial directions (5/3). The obtained subbands of three approaches are coded with 3D SPIHT.

The lifting scheme is very applied in medical image compression with traditionnel filters as $5 / 3$ but other new filters can be tested in spatial directions [16]. The motion vectors are determined by block motion compensation model with full search method, the search is effectuated with block size of $16 \times 16$ pixels in our approach and MC Trunc 5/3 approach. The Table 1 lists the obtained average bit rate for the lossless compression using two first volumes of each 4D medical image.

Table 1. 4D Lossless Compression Results in bit per pixel (bpp)

\begin{tabular}{llll}
\hline 4D Medical Images & 3D SPIHT & MC Trunc 5/3 & Proposed Method \\
\hline Image1 & 5.19 & 5.23 & 5.10 \\
Image2 & 5.92 & 5.97 & 5.89 \\
Image3 & 5.39 & 5.45 & 5.36 \\
Image4 & 5.03 & 5.06 & 4.98 \\
Image5 & 4.53 & 4.55 & 4.35 \\
\hline
\end{tabular}

The results show our method outperforms the other methods for all test images. 3D SPIHT gives low average bit rate than $\mathrm{MC}$ Trunc $5 / 3$ of $0.76 \%$, thus the integration of the motion compensated truncated $5 / 3$ lifting filter in 3D SPIHT penalises the compression rate in MC Trunc 5/3 approach. However the proposed inter slices filter improves the compression rate (low bit rate) with average of $1.45 \%$ compared to 3D SPIHT, as well a net improvement is reached of the order of $2.2 \%$ compared to MC Trunc 5/3.

The average improvement by proposed technique compared to 3D SPIHT is obtained by using image 1 thus we use it in following test. The experimental results presented in Table 2 are obtained by testing five different sizes of image1, thus line 2 represents lossless compression results of 2 first volumes of image 1 , line 3 of 4 first volumes of Image 1 and so on to the last line of 10 first volumes of image1, these different sizes of Image 1 permit to obtain differents GOS by reorganizing the slices as in Figure 2 (b).

Table 2. 4D Lossless Compression Results in bit per pixel (bpp) for different sizes of Image1

\begin{tabular}{llll}
\hline 4D Medical Images & 3D SPIHT & MC Trunc 5/3 & Proposed Method \\
\hline 2Volumes Image1 & 5.19 & 5.23 & 5.10 \\
4Volumes Image1 & 5.08 & 5.10 & 4.99 \\
6Volumes Image1 & 5.07 & 5.05 & 4.96 \\
8Volumes Image1 & 4.98 & 5.00 & 4.92 \\
10Volumes Image 1 & 4.99 & 4.96 & 4.90 \\
\hline
\end{tabular}

The obtained compression rate by proposed method is better than other schemes for all images test. We note that the bit rate is reduced for all compression schemes while the number of temporal slices (at different time and with the same position $z$ ) composed GOS increase. The results show a variation of the compression rate as a function of the number of volumes. The improvement of this rate by our approach is of the order of $1.73 \%$ and $1.85 \%$ compared to 3D SPIHT and MC Trunc 5/3 respectively, while it does not exceed $0.11 \%$ between the latter two methods.

\section{CONCLUSION}

In this paper we proposed a new approach of lossless compression of 4D medical images, it consists in construction of GOS and each slices composed GOS is decorrelated in spatial directions $(x, y)$, after proposed inter slices filtering is performed with minimum of three predicted errors for each block composed inter slices. The three prediction errors are represented by unfiltered block, truncated 5/3 lifting filter and motion compensated the aim is to reduce the size of residual slices. The obtained slices are coded with 3D SPIHT. The proposed approach provides lossless compression improvements by reducing the bit rates compared to 3D SPIHT with MC trunc 5/3 and 3D SPIHT without motion compensation, this enhancement is due to the integration of the three prediction techniques in inter slices filter. As future work, our approach offers the possibility to obtain lossy to lossless decoding by exploiting inter slices scalability also other 4D medical image modalities can be tested. 


\section{REFERENCES}

[1] V. Sanchez, P. Nasiopoulos, R. Abugharbieh, "Lossless Compression of 4D Medical Images using H.264/AVC, " IEEE International Conference on Acoustics, Speech, and Signal Processing, Toulouse, 2006, pp. 1116-1119.

[2] V. Sanchez, P. Nasiopoulos, R. Abugharbieh, "Efficient lossless compression of 4D medical images based on the advanced video coding scheme," IEEE Trans. Information Technology In Biomedicine, Vol. 12, NO. 4, JULY 2008, pp. 132-138.

[3] U. Martin, A. Kaup, "Analysis of compression of $4 D$ volumetric medical image datasets using multi-view (MVC) video coding methods," Mathematics of Data/Image Pattern Recognition, Compression and Encryption with Applications XI, Proc. of SPIE. Vol. 7075, 70757,2008.

[4] Y. Feng, H. Lu, X. Zeng, "A Fractal Image Compression Method Based on Multi-Wavelet", TELKOMNIKA (Telecommunication, Computing, Electronics and Control), Vol.13, No.3, September 2015, pp. 996-1005.

[5] Y. Habchi, M. Beladgham, A. A. Taleb , "RGB Medical Video Compression Using Geometric Wavelet and SPIHT Coding ", International Journal of Electrical and Computer Engineering (IJECE), Vol. 6, No. 4, August 2016, pp. 1627-1636.

[6] H. G. Lalgudi, A. Bilgin, M. W. Marcellin, A. Tabesh, M. D. Nadar and T. P. Trouard, "Four-dimensional compression of fMRI using JPEG2000," Medical Imaging 2005:Image Processing, Proc. of SPIE. Vol. 5747.

[7] A. A. Kassim, P. Yan, W. S. Lee, K. Sengupta, "Motion Compensated Lossy-to-Lossless Compression of 4D Medical Images Using Integer Wavelet Transforms," IEEE Trans. Information Technology In Biomedicine, VOL. 9, NO. 1, 2005, pp. 132-138.

[8] J. -R. Ohm, "Three-dimensional subband coding with motion compensation," IEEE Trans. on Image Processing, 1994, pp. 559-571.

[9] S. -J. Choi and J. Woods, "Motion-compensated 3-d subband coding of video," IEEE Trans. on Image Processing, 1999, pp. 155-167.

[10] A. Secker and D. Taubman, "Motion-compensated highly scalable video compression using an adaptative $3 \mathrm{~d}$ wavelet transform based on lifting," IEEE, 2001.

[11] L. Luo, J. Li, S. Li, Z. Zhuang, and Y-Q .Zhang, "Motion-compensated lifting wavelet and its application in video coding," in IEEE International Conference on Multimedia and Expo, August 2001.

[12] I. Daubechies and W. Sweldens, "Factoring wavelet transforms into lifting steps," Journal of Fourier Analysis and Applications, 1998.

[13] B. J. Kim and W. A. Pearlman, "An Embedded Wavelet Video Coder Using Three-Dimensional Set Partitioning in Hierarchical Trees (SPIHT)," In IEEE Data Compression Conference, 1997, pp. 221-260.

[14] J. Vandemeulebroucke, S. Rit, J. Kybic, P. Clarysse, and D. Sarrut, "Spatiotemporal motion estimation for respiratorycorrelated imaging of the lungs," In Med Phys, 2011, pp. 166-178.

[15] http://midas.kitware.com/community/view/47.

[16] A. Hazarathaiah, B. Prabhakara Rao, "Medical Image Compression using Lifting based New Wavelet Transforms", International Journal of Electrical and Computer Engineering (IJECE), Vol. 4, No. 5, October 2014, pp. 741-750. 